\title{
ORDER-CLUSTERED FIXED POINT THEOREMS AND THEIR APPLICATIONS TO PARETO EQUILIBRIUM PROBLEMS
}

\author{
LINSEN XIE* ${ }^{*}$ JINLU LI**, ADRIAN PETRUŞEL*** AND JEN-CHIH YAO**** \\ * Department of Mathematics, Lishui University, Lishui, Zhejiang 323000, China \\ E-mail: linsenxie@163.com \\ ${ }^{* *}$ Department of Mathematics, Shawnee State University, Portsmouth, Ohio 45662, USA \\ E-mail: jli@shawnee.edu \\ ***BBabeş-Bolyai University, Cluj-Napoca, Romania \\ E-mail: petrusel@math.ubbcluj.ro \\ **** Center for General Education, China Medical University, Taichung 40402, Taiwan \\ E-mail: yaojc@mail.cmu.edu.tw
}

\begin{abstract}
In this paper, we provide some properties of order clusters in preordered sets and we prove some order-clustered fixed point theorems on preordered sets. Then by applying these theorems, we show the existence of ordered Pareto equilibrium and Nash equilibrium for some noncooperative strategic games with incomplete (preordered) preferences.

Key Words and Phrases: Chain-complete preordered sets, order-clustered fixed point, fixed point, Pareto equilibrium, Nash equilibrium.
\end{abstract}

2010 Mathematics Subject Classification: 46B42, 47H10, 91A06.

Acknowledgements. The first author was partially supported by the National Natural Science Foundation of China (11171137). The fourth author was partially supported by the Grant MOST 103-2923-E-039-001-MY3.

\section{REFERENCES}

[1] C.D. Aliprantis, O. Burkinshaw, Positive Operators, Springer, 2006.

[2] H.F. Bohnenblust, S. Karlin, Contributions to the Theory of Games, Princeton Univ. Press, 1950.

[3] S. Carl, S. Heikkila, Fixed Point Theory in Ordered Sets and Applications: From Differential and Integral Equations to Game Theory, Springer, New York, 2010.

[4] N. Dunford, J.T. Schwarts, Linear Operators, Part I, John Wiley and Sons, 1988.

[5] T. Fujimoto, An Extension of Tarski's fixed point theorem and its applications to isotone complementarity problems, Math. Programming, 28(1984), 116-118.

[6] N.J. Huang, Y.P. Fang, On vector variational inequalities in reflexive Banach spaces, J. Global Optim., 32(2005), 495-505. 
[7] F. Giannessi, Theorems of Alterative, Quadratic Programs and Complementarity Problems, Edited by Cottle, R.W., Giannessi, F. and Lions, J.L., John Wiley and Sons, Chi Chester, 1980, 151-186.

[8] J.L. Li,Several extensions of the Abian-Brown fixed point theorem and applications to generalized and extended Nash equilibria on chain-complete posets, J. Math. Anal. Appl., 409(2014), 1084-1092.

[9] J.L. Li, Inductive properties of fixed point sets of mappings on posets and on partially ordered topological spaces, Fixed Point Theory Appl., 2015(2015):211

[10] J.L. Li, Applications of fixed point theory to generalized Nash equilibriums of non-monetized non-cooperative games on Banach lattices, Nonlinear Anal. Forum, 18(2013), 1-11.

[11] J.L. Li, On the Pareto equilibrium problems with partially ordered preferences, Nonlinear Anal. Forum, 21(2016) no. 1, 13-22.

[12] E.A. Ok, Order Theory, (forthcoming).

[13] J. Von Neumann, O. Morgenstern, The Theory of Games and Economic Behavior, Princeton University Press, 1944.

[14] L.S. Xie, J.L. Li, W.S. Yang, Order-clustered fixed point theorems on chain-complete preordered sets and their applications to extended and generalized Nash equilibria, Fixed Point Theory Appl., 2013(2013):192.

[15] C.J. Zhang, Set-valued Analysis with Applications in Economics (Chinese), Sciences Press, Beijing, 2004

Received: June 26, 2015; Accepted: January 10, 2016. 